\title{
A DYNAMO THEORY PREDICTION FOR SOLAR CYCLE 22: SUNSPOT NUMBER, RADIO FLUX, EXOSPHERIC TEMPERATURE, AND TOTAL DENSITY AT $400 \mathrm{KM}$
}

\author{
K. H. Schatten and A. E. Hedin \\ Laboratory for Atmospheres \\ NASA/Goddard Space Flight Center \\ Greenbelt, MD 20771
}

Using the "dynamo theory" method to predict solar activity, a value for the smoothed sunspot number of $109 \pm 20$ is obtained for solar cycle 22 . The predicted cycle is expected to peak near December, $1990 \pm 1$ year. Concommitantly, $F_{10.7}$ radio flux is expected to reach a smoothed value of $158 \pm 18$ flux units. Global mean exospheric temperature is expected to reach $1060 \pm 50 \mathrm{~K}$ and global total average total thermospheric density at $400 \mathrm{~km}$ is expected to reach $4.3 \times 10^{-15} \mathrm{gm}$ $\mathrm{cm}^{-3} \pm 25 \%$. 


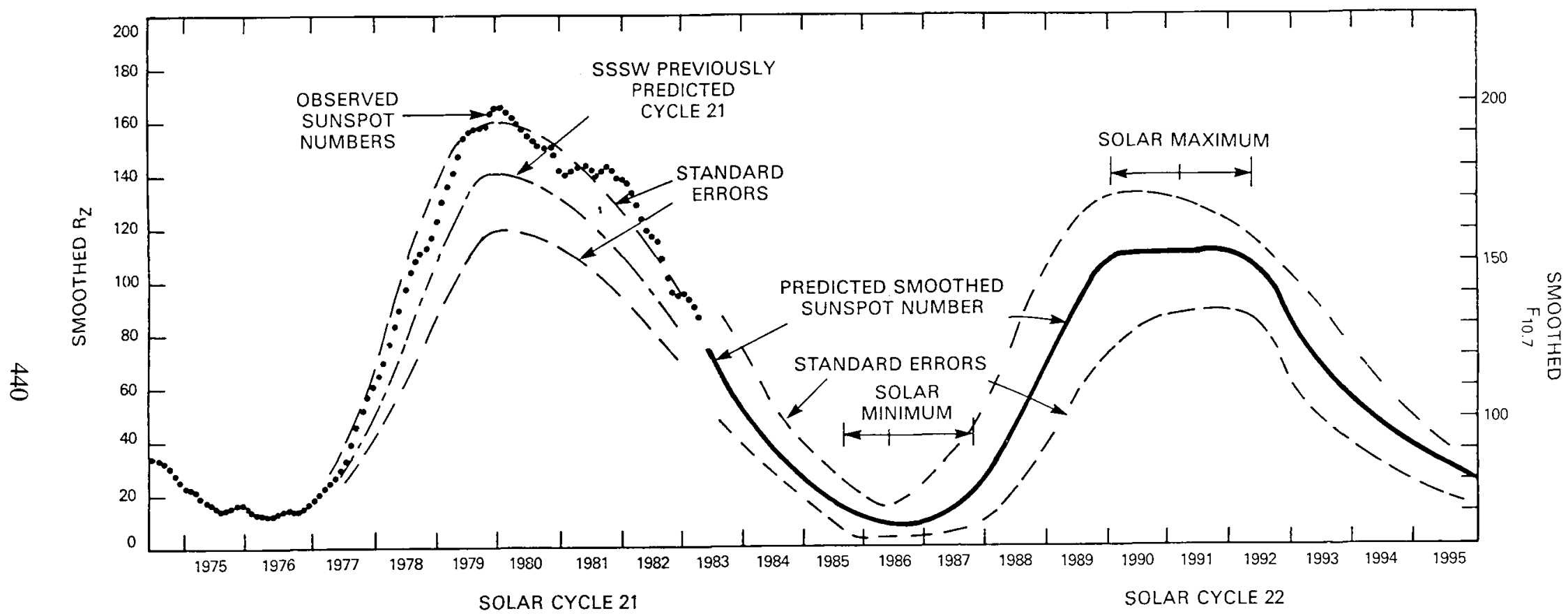

Figure 1. Predicted smoothed sunspot number and radio flux from 1984 to 1995 (solid curve). Estimated early average uncertainties (dashed curves) are smaller than in many other predictions. The previous prediction for solar cycle 21 (shown) compares well with the observed sunspot numbers (dots). 

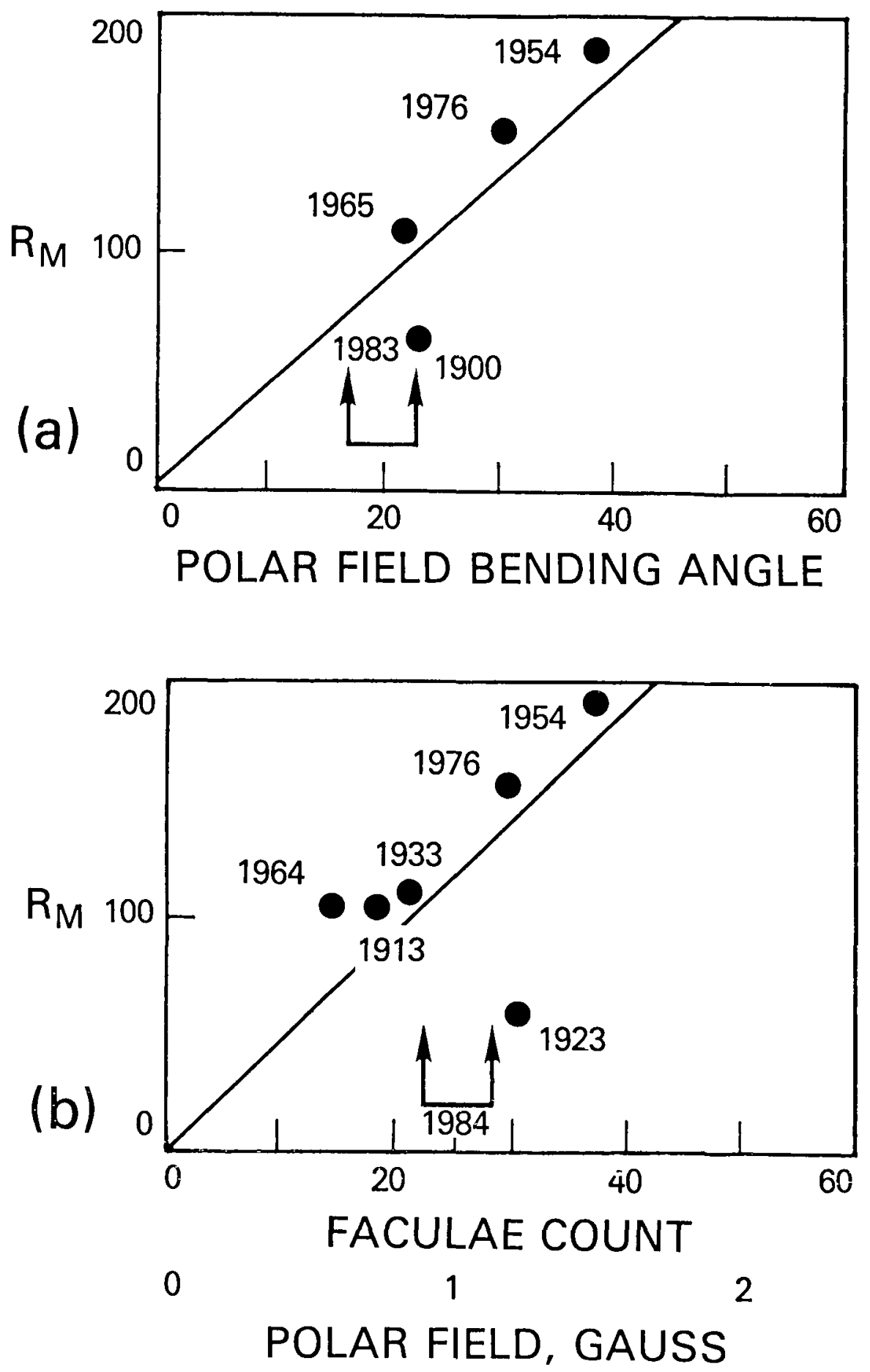

Figure 2. Smoothed maximum sunspot numbers versus polar field bending angle (a) and field strength and faculae count (b) at or near the previous sunspot minimum. The dots are labeled by the year that the polar field value was obtained. The arrows show the 1983 (top) value for the polar field bending and the 1984 (bottom) polar field strength. 


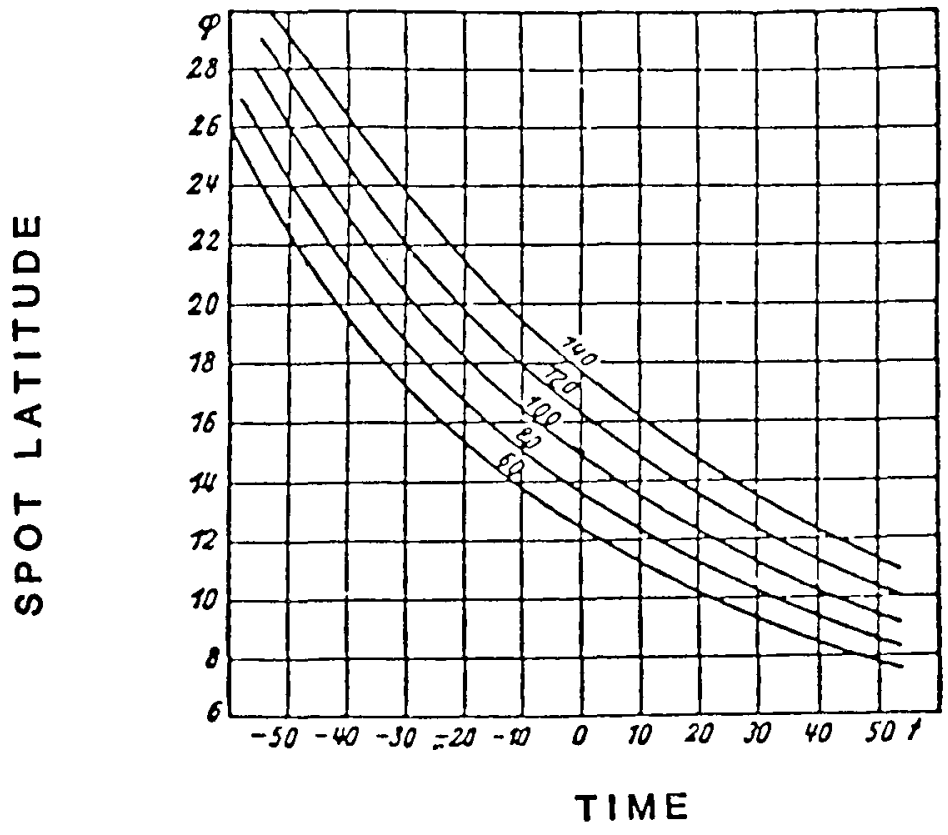

Figure 3. Migration of spot zone for different $\mathbf{R}_{\mathrm{m}}$ (heights of sunspot maximum). Abcissae: time measured in solar rotation from sunspot maximum; ordinates: latitude (Waldmeier, 1939). 


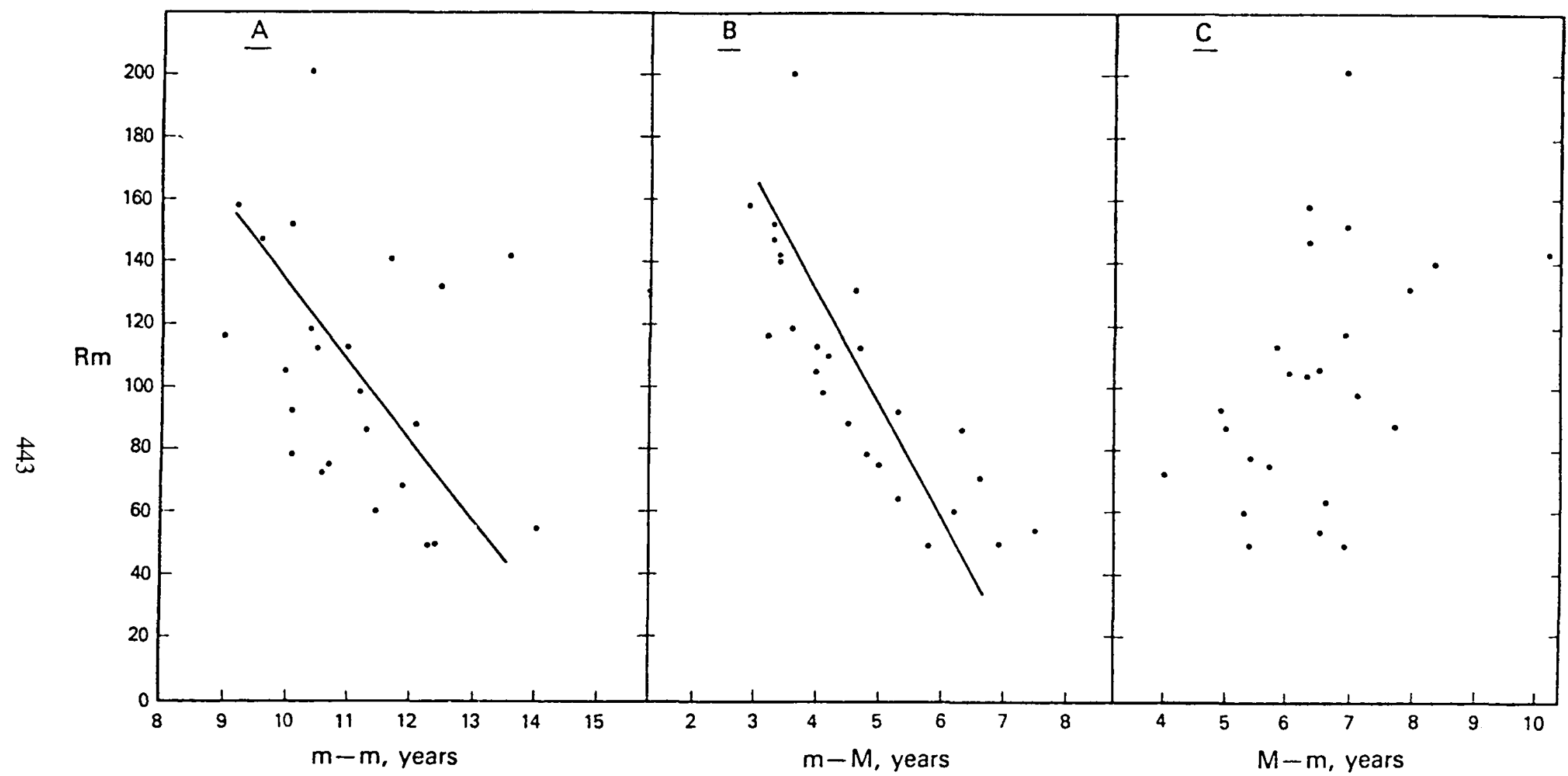

Figure 4(a). Left-The solar activity minimum to minimum duration $(\mathrm{m}-\mathrm{m})$, is graphed vs. solar maximum sunspot number. (b) Middle-The rising portion of the cycles' duration (m-M) is graphed. (c) Right-The trailing end of the sunspot cycles' duration (M-m). 


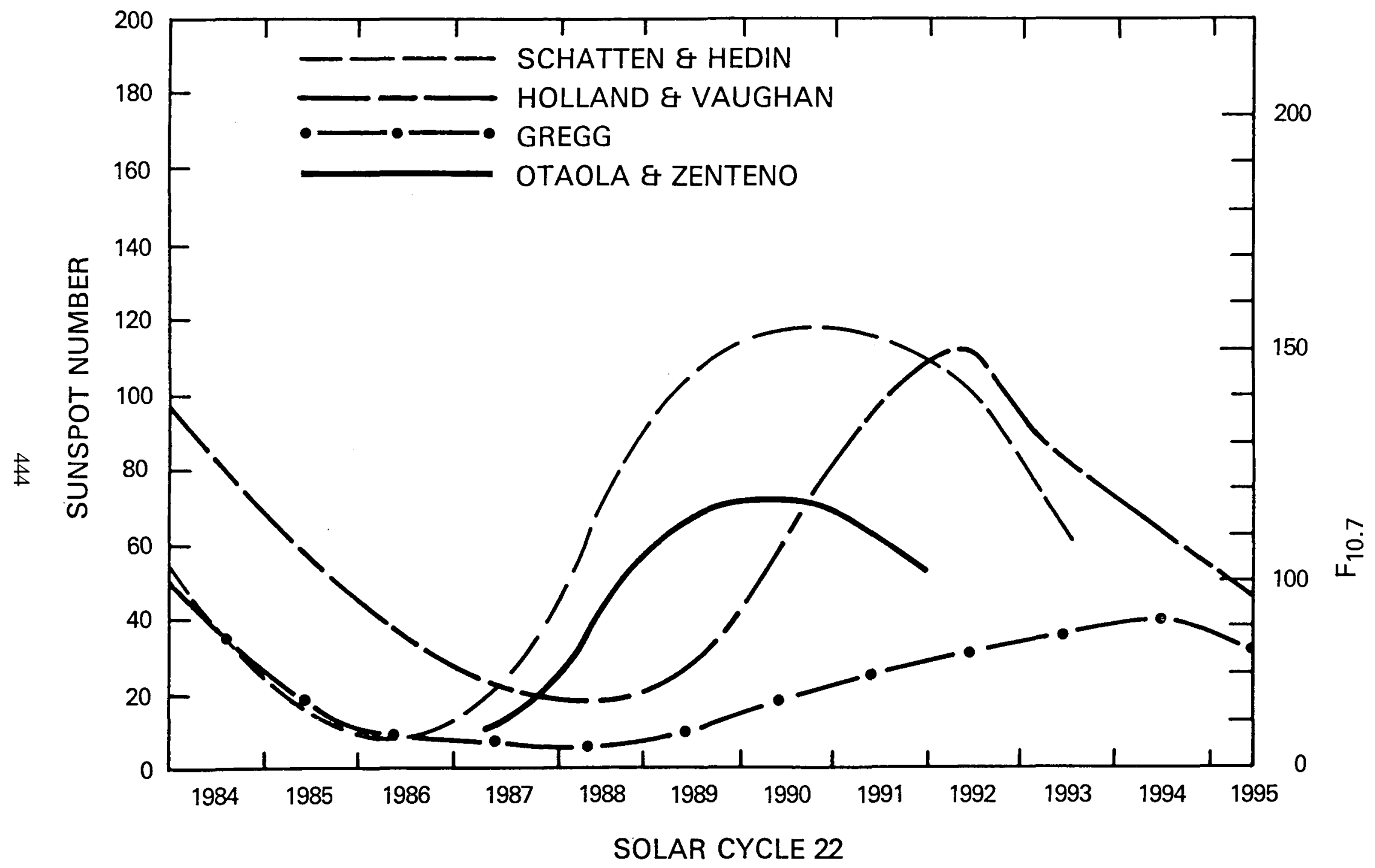

Figure 5. Shown are the predicted sunspot numbers from 1984 to 1995 with various authors' methods. 